\title{
Rapid preparation of all-cellulose composites by solvent welding based the use of aqueous solvent
}

Juho Antti Sirviö, ${ }^{a *}$ Miikka Visanko ${ }^{\text {a }}$, Nils C. Hildebrandt ${ }^{\mathrm{a}, \mathrm{b}}$

${ }^{a}$ Fibre and Particle Engineering Research Unit, University of Oulu, P.O. Box 4300, 90014 Oulu, Finland

${ }^{\mathrm{b}}$ Baden-Wuerttemberg Cooperative State University, Erzbergerstraße 121, 76133 Karlsruhe, Germany

Keywords: Cellulose dissolution, tetraalkylammonium hydroxide, all-cellulose composite, mechanical properties

\begin{abstract}
In the present study, the dissolution of softwood fibers and fabrication of all-cellulose composites was investigated using aqueous tetraethylammonium hydroxide solution (TEAOH, $35 \mathrm{wt} \%)$ at room temperature. The bulk of the cellulose fibers were instantly dissolved when mixed with the solvent, and suspensions up to $3 \mathrm{wt} \%$ were fabricated with ease. During the dissolution, a few remaining larger fiber flocks were fully dissolved within $3 \mathrm{~h}$ of mixing at room temperature. TEAOH was further studied in the production of all-cellulose composites by solvent welding (partial dissolution) of cellulose fiber sheets. Tensile strength of the original cellulose sheet increased from 3.3 to $55.0 \mathrm{MPa}$ only after $30 \mathrm{~s}$ of room-temperature solvent welding, and an increase of over 23 -fold (up to $76.7 \mathrm{MPa}$ ) was obtained by high-pressure-aided hot-pressing of the partially dissolved sheets before drying. The demonstrated fabrication of allcellulose composites yielded many benefits in comparison to the current dissolution systems,
\end{abstract}


such as short treatment time, ambient operation conditions, and readily applicable processing solutions, which makes it a potential methodology applicable even at an industrial scale.

\section{Introduction}

Cellulose is the most abundant organic polymer on earth, and its broad availability makes it a promising raw material for novel applications.[1] Chemical modification or solvent-based shaping (including dissolution) of cellulose can broaden its properties, as required by various applications.[2,3] However, owing to its hydrogen bonding interaction and high crystallinity, cellulose has poor solubility in most common solvents.[4] Therefore, novel dissolution methods of cellulose have high importance in the field of sustainable chemistry and materials fabrication.

Cellulose can be dissolved using chemical derivatization (e.g., esterification[5] or carbamation[6,7]). However, these systems change the chemical and physical properties of cellulose. Cellulose can also be dissolved in various non-derivatizing solvent systems, including N-methylmorpholine-N-oxide (NMMO)[8], dimethylacedamide/LiCl[9], and molten salt hydrates[10]. However, these systems exhibit certain disadvantages, such as solvent instability, degradation of cellulose, and high toxicity. Ionic liquids are currently one of the most utilized solvents for cellulose dissolution.[11,12] Some novel ionic liquids and organic solvent mixtures (termed as organic electrolyte solutions) have been shown to rapidly dissolve cellulose.[13] With respect to sustainability, aqueous alkaline (sodium or lithium) hydroxides together with urea or thiourea are some of the most promising cellulose solvents.[14-16] Minor disadvantages of these systems include the requisite for dissolution at a chilled temperature of $-12^{\circ} \mathrm{C}[17]$ and limited capacity to dissolve high-molecular-weight cellulose.[18] Recently, tetra-alkylammonium hydroxides were shown to efficiently dissolve cellulose even at room temperature (however, the dissolution of high-molecular-weight cellulose generally requires elevated temperatures).[19-21] 
Despite the apparent toxicity of alkylammonium cations, the recyclability makes tetraalkylammonium hydroxides a suitable solvent for isolation of cellulose originating from different sources.[20,22]

All-cellulose composites (ACCs) are a relatively new class of cellulosic material, in which dissolved cellulose is utilized as a matrix to bond together natural cellulose fibers.[2] ACCs can be produced by mixing together dissolved cellulose and cellulose fibers (supposing that fibers do not dissolve during the processing) followed by casting and regeneration. Another way is solvent-based modification of cellulose sheets by partial dissolution and regeneration (referred as surface selective dissolution[23], partial fibre dissolution[24], or solvent welding[25]). Several different solvent systems have been studied for solvent welding of cellulose fibers, including dimethyl acetamide/LiCl[24], $\mathrm{NMMO}$ [26], ionic liquids[27], or $\mathrm{NaOH}$-urea[28,29]. With these systems, ACCs were successfully produced with good properties, but they all pose some shortcomings, including long dissolution times and requirement of either high (e.g., $\left.80{ }^{\circ} \mathrm{C}\right)$ or low temperatures $\left(<0{ }^{\circ} \mathrm{C}\right)$. Use of high or low temperature can be environmentally desirable due to the high energy consumption, in addition to increase of the product cost. Especially from an industrial point of view, the dilatory dissolution of cellulose can be challenging to implement if a continuous process operation is desired.

In this study, the dissolution of high-molecular-weight cellulose fibers from dissolving pulp was studied using aqueous tetraethylammonium hydroxide (TEAOH, $35 \mathrm{wt} \%$ in water). The effect of dissolution and regeneration on the degree of polymerization (DP) and crystallinity of cellulose were studied using limiting viscosity and wide-angle X-ray diffraction (WAXD), respectively. ACCs were produced based on the rapid dissolution efficiency of TEAOH by solvent welding cellulose sheets. The effect of the dissolution time and hot-pressing on the 
surface morphology and mechanical properties of ACCs were investigated by scanning electron microscopy (SEM) and tensile testing, respectively.

\section{Material and Methods}

\section{Materials}

Cellulose dissolving pulp (softwood) was obtained as dry sheets, whose properties are presented elsewhere.[30] TEAOH (35\% in water) was obtained from Sigma Aldrich (Germany). Ethanol and $\mathrm{HCl}(0.1 \mathrm{M})$ were acquired from VWR (Finland).

\section{Dissolution of cellulose}

Dissolving pulp sheets were disintegrated in water using standard procedure [31] followed by filtration, washing with technical ethanol for $30 \mathrm{~min}$, and filtration again before drying in an oven $\left(60{ }^{\circ} \mathrm{C}\right)$. Fibers were then dissolved in $35 \mathrm{wt} \%$ TEAOH in water by mixing the fibers directly in the solvent using magnetic stirrer or by adding the solvent to the fibers $(0.5-3 \mathrm{wt} \%$ cellulose solutions) while simultaneously mixing at room temperature. After solvent addition, the suspensions were mixed for $3 \mathrm{~h}$ until all the fibers were dissolved, and the solubility was verified using an optical microscope. Cellulose was regenerated by pouring the solution into ethanol while mixing. Regenerated cellulose was filtered and washed using ethanol until the washing liquid was neutral (checked using $\mathrm{pH}$ paper).

\section{Fabrication of all-cellulose composites}

Laboratory hand sheets $\left(80 \mathrm{~g} / \mathrm{m}^{2}\right)$ were prepared from the dissolving pulp, according to a standard procedure to produce sheet with thickness around $180 \mu \mathrm{m} .[32]$ Around $5 \mathrm{~cm} \mathrm{x} 7 \mathrm{~cm}$ pieces were cut from the laboratory sheets and immersed in excess of TEAOH for 30,60, 120, or $180 \mathrm{~s}$ for solvent welding of the fibers. No mixing of the solvent applied. The sheets were then 
carefully lifted from the solvent and placed in $0.1 \mathrm{M} \mathrm{HCl}$ solution for $120 \mathrm{~s}$. After acid coagulation, ACCs were first washed in a beaker containing deionized water $(500 \mathrm{ml})$ for $30 \mathrm{~min}$ and then rinsed under running deionized water until the surfaces were neutral (checked using $\mathrm{pH}$ paper). Two different drying procedures were applied in the finalization of ACCs fabrication. The ACCs were placed between a filter paper and hand sheet on both sides and dried either in a drying grill (temperature of plates was around $125^{\circ} \mathrm{C}$ ) for $5 \mathrm{~min}$ or under high-pressure and heat (high-pressure aided hot-pressing, hp) using the Fontune Press (the Netherlands). In the latter, two consecutive steps were applied: 1) the sample was dried using a force of $45 \mathrm{kN}$ together with the filter papers. 2) The dried ACC without filter papers was placed between the hot metal plates and pressed with a force of $300 \mathrm{kN}$ to remove possible residual moisture. The temperature in both steps was $150^{\circ} \mathrm{C}$.

\section{Attenuated total reflection infrared spectroscopy}

The chemical characterization of raw, regenerated, and ACCs was performed using an ATRIR. The spectra were collected from dried samples using a Bruker Tensor II FT-IR Spectrometer with a Hyperion 3000 FT-IR Microscope (USA). Spectra were obtained in the $600-4000 \mathrm{~cm}^{-1}$ range, and 40 scans were taken at a resolution of $4 \mathrm{~cm}^{-1}$ for each sample.

\section{Degree of polymerization}

The average degree of polymerization (DP) of the dissolving cellulose pulp and regenerated cellulose were evaluated using the limiting viscosity number, as measured in the CED solution according to the ISO 5351 standard. The samples were freeze-dried prior to the measurement. The limiting viscosity numbers were converted to DP using Eq. (1), 


$$
D P=\left(\frac{(1.65[\mathrm{\eta}]-116 H}{C}\right)^{1.111}
$$

where $[\eta]$ is the limiting viscosity number, $\mathrm{C}$ is the mass fraction of the cellulose, and $\mathrm{H}$ is the mass fraction of the hemicelluloses. This calculation corrects for the contribution of the hemicelluloses to the limiting viscosity number and DP of the cellulose, assuming that the average DP of the hemicelluloses is 140 .

\section{Mechanical properties}

The tensile tests were performed using a universal material testing machine (Instron 5544, USA) equipped with a $100 \mathrm{~N}$ load cell. The laboratory sheet from the dissolving pulp and ACCs were cut into thin strips with a specimen width of $5 \mathrm{~mm}$, and their thicknesses were measured using a precision thickness gauge (Hanatek FT3, UK). For the tensile testing, a 40-mm gauge length was set under a strain rate of $4 \mathrm{~mm} / \mathrm{min}$ and six specimens were measured. The tests were conducted in $50 \% \mathrm{RH}$ at a temperature of $23{ }^{\circ} \mathrm{C}$ and under a pre-load of $0.05-0.1 \mathrm{~N}$. Prior to testing, the specimens were conditioned for at least one day in the same environment. The elastic modulus was calculated from the initial linear portion of the stress-strain curve, and the ultimate tensile strength was defined as the stress at the specimen breakage. At least five specimens from each samples were measured and specimens flailed at the clamps were excluded.

\section{X-ray diffraction}

The crystalline structures of the dissolving pulp, regenerated cellulose, and ACCs were investigated using WAXD. Measurements were conducted on a Rigaku SmartLab 9kW rotating anode diffractometer (Japan) using a Co Ka radiation $(40 \mathrm{kV}, 135 \mathrm{~mA})(\lambda=1.79030 \mathrm{~nm})$. Samples from the dissolving pulp and regenerated cellulose were prepared by pressing tablets of 
freeze-dried celluloses to a thickness of $1 \mathrm{~mm}$. ACCs were measured similarly. Scans were taken over a $2 \theta$ (Bragg angle) range from $5^{\circ}-50^{\circ}$ at a scanning speed of $10^{\circ} / \mathrm{min}$, using a step width of $0.5^{\circ}$. The degree of crystallinity was determined by the method of Nam et al.[33] with the modifications suggested by Piltonen et al.[29] Since it is known from the literature that regenerated cellulose tends to have a preferred orientation, the March-Dollase parameter for the (11̄0)-plane in cellulose II was adjusted in the simulated patterns for each sample to find the best

matching fit.[34] The March-Dollase parameter was then converted to the degree of preferred orientation of cellulose II using Eq. 2,[35]

$\eta_{P O}=100 \% \cdot \sqrt{\frac{(1-r)^{\mathrm{a}}}{1-r^{3}}}$

where $\eta_{P O}$ is the degree of the preferred orientation, and $r$ is the March-Dollase parameter.

\section{Scanning Electron Microscopy}

SEM (Zeiss Zigma HD VP, Germany) images of ACCs and untreated reference dissolving pulp sheet were obtained using Zeiss Zigma HD VP (Germany), with an accelerating voltage of $5 \mathrm{kV}$ during imaging. Prior to the measurement, the samples were placed on carbon tape and sputter coated with Pt for 30 second using one coating cycle.

\section{Results and Discussion}

\section{Dissolution of cellulose in tetraethylammonium hydroxide solution}

Prior the ACC production, the efficiency of TEAOH on the cellulose dissolution at room temperature was investigated. Most of the cellulose fibers dissolved completely in TEAOH (35 
$\mathrm{wt} \%$ in water) immediately after coming into contact with the solvent. However, some of the fiber bundles or "chunks" were difficult to dissolve, and complete dissolution was achieved after $3 \mathrm{~h}$ of mixing at room temperature (Figure $1 \mathrm{~b})$. The completely dissolution (1-3\%) was dependent on the mixing speed and cellulose concentration (Figure 1c and Figure S1). The formation of less soluble chunks in the solvent were noted to occur when bigger shreds of fibers were added into the solvent. The gelation of the outer layer of the less soluble shreds hindered the diffusion of the solvent. Similar behavior is encountered when methyl cellulose is dissolved in cold water.[36] One way to improve fiber dissolution and minimize the formation of gelcoated chunks was to rapidly add the solvent on the top of the fiber network for improved penetration while simultaneously mixing. The $3 \%$ solution was easily obtained at room temperature, however, due to the high viscosity, no attempt to produce higher concentration solution was performed as the aim of the current study was to investigate the preparation of ACCs at room temperature. Higher solution concentrations could be achieved combining more intensified mixing or by applying higher temperature.
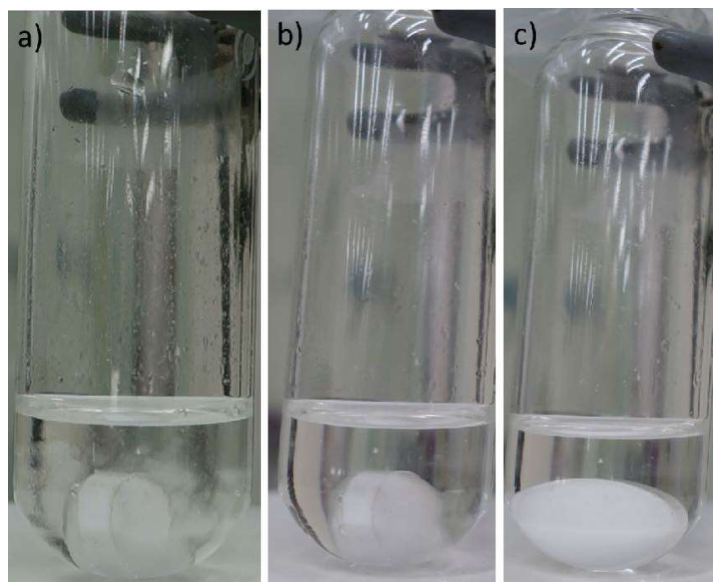

Figure 1 a) $1 \%$ cellulose solution in TEAOH ( $35 \mathrm{wt} \%$ in water) a) after addition of solvent, and b) after one hour, and c) after three hours under mixing. 
After dissolution, cellulose could be regenerated by the addition of ethanol as an anti-solvent (or be using acid such as dilute $\mathrm{HCl}$ solution, which was used when in the production of ACCs). DP of the cellulose remained intact after regeneration. Mild dissolution conditions $(3 \mathrm{~h}$ at room temperature) might help prevent the unwanted hydrolysis of cellulose as the initial DP of dissolving pulp remained at 1862 after regeneration. The yield of the regenerated cellulose was over $90 \%$, and it is most likely that the yield lost is towing to the sample handling and the possible removal of residual hemicelluloses $(<6 \%)$ in the pulp. In addition, based on the elemental analysis, all the TEAOH was removed after washing.

Previous studies have indicated poor solubility of cellulose in aqueous TEAOH (Avicel PH101 cellulose powder).[19] In the current study $35 \mathrm{wt} \%$ TEAOH solution obtained from Sigma Aldrich exhibited good dissolution capacity towards cellulose. However, in our studies we observed with similar solution obtained from different supplier (TCI Chemicals) poor dissolution capacity. According to the supplier, some TEAOH solutions might contain bromide-ion as impurity (TEAOH is obtained from corresponding bromide salt), which might alter the properties of solvent. Previously it has been observed that impurities, such as alkaline metal ions can hinder the dissolution of cellulose in tetrabutylammonium hydroxide.[37] Therefore, further studies are needed to investigate the exact effect of the impurities on the dissolution of cellulose in TEAOH.

\section{Fabrication and characterization all-cellulose composites}

The laboratory sheets were prepared from the dissolving pulp, and ACCs were fabricated by room-temperature welding of fibers using TEAOH. Visual shrinking of the cellulose sheet was observed immediately after submersion into TEAOH. Partially transparent ACC hydrogel was obtained after coagulation in an acid bath $(0.1 \mathrm{M} \mathrm{HCl})$ and washing with deionized water. $\mathrm{HCl}$ 
was used to ensure rapid coagulation of cellulose, however, further studies should be conducted to verify the effect of coagulation bath on the properties of ACCs. All of the samples could be easily handled and washed under running water after coagulation. After drying, the ACCs After drying, the ACCs appeared relatively transparent when placed on top of the background (Figure 2a), resembling those previously obtained by dimethyl acetamide/LiCl[23,38], which, however, required several hours for partial dissolution of cellulose. However, The haziness was apparent when stripes from the ACCs were held one centimeter above the background image (Figure $2 b$ ).

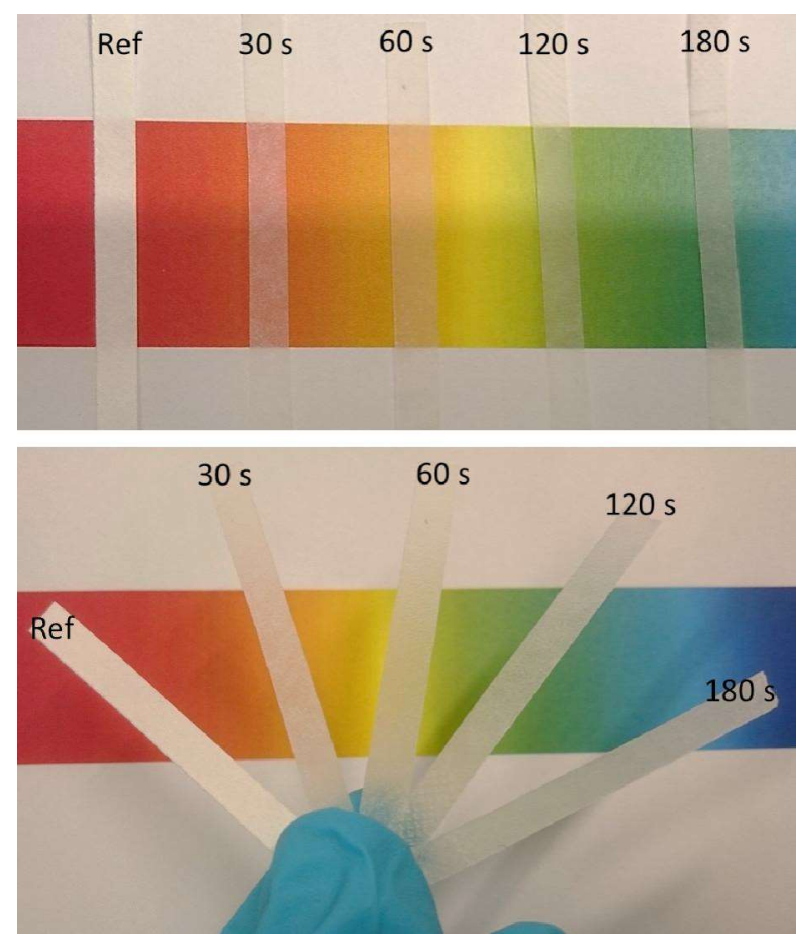

Figure 2. Visual appearance of reference cellulose sheet and ACCs after various treatment times directly on the top of (upper photo) and around one centimeter above (lower photo) the background demonstrating the semi-transparency of ACCs.

The ATR-IR spectrum of the original dissolving pulp (Ref), completely dissolved and regenerated dissolving pulp (Reg), and ACC after $30 \mathrm{~s}$ treatment (30s) are presented in Figure 4 
(similar spectra were acquired for the other ACCs and spectra are presented in Figure S2 in Supporting information). Several alterations in the spectrum of the ACC compared to that of the dissolving pulp indicates the partial transformation of cellulose I crystalline structure to ether cellulose II crystalline structure or amorphous cellulose.[34] The peak in the -OH region (around $3300 \mathrm{~cm}^{-1}$ ) was broadened, and new shoulders appeared around 3480 and $3440 \mathrm{~cm}^{-1}$ (Figure 3a). The flattening and the change in the peak maximum from 1427 to $1421 \mathrm{~cm}^{-1}$ was related to symmetric $\mathrm{CH}_{2}$ bending or scissoring motions in cellulose I, further indicating the transformation of cellulose I to cellulose II crystalline structure or to amorphous cellulose during the ACC preparation (Figure 3b). The clear separation of the 1313 and $1336 \mathrm{~cm}^{-1}$ peaks, related to the $\mathrm{CH}_{2}$ wagging motion and to the $-\mathrm{OH}$ in-plane bending of the crystalline forms of cellulose $\mathrm{I}$ and cellulose II, respectively, indicates that cellulose was not entirely transformed to amorphous or poorly crystalline cellulose. In contrast, the regenerated cellulose exhibited a broader peak at $3300 \mathrm{~cm}^{-1}$ compared to the original fibers and ACC. In addition, a broad peak can only be observed at around $1420 \mathrm{~cm}^{-1}$, and the absence of a clear peak at $1313 \mathrm{~cm}^{-1}$ indicates that the dissolution and regeneration of the dissolving pulp converts cellulose I to cellulose II and to amorphous or poorly crystalline cellulose. Differences in the ATR-IR spectra of ACCs and regenerated cellulose is most likely due to the use of different coagulation bath, which can cause differences in the crystallinity and surface properties of cellulose. [39,40] 

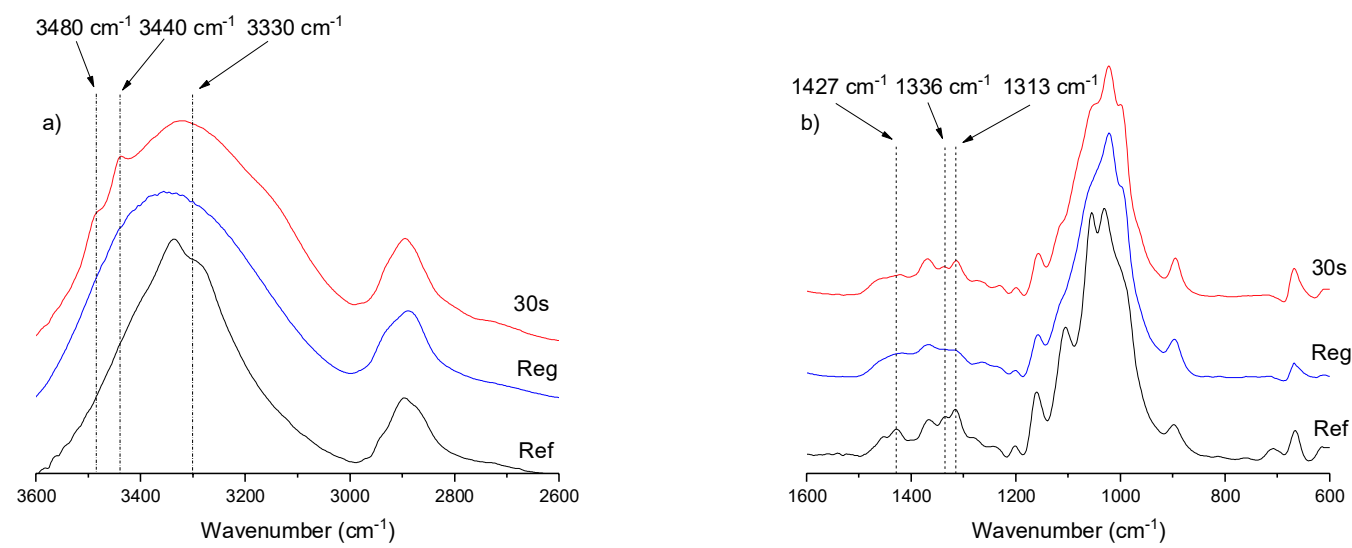

Figure 3. ATR-IR spectra of original cellulose pulp (Ref), completely dissolved and regenerated cellulose (Reg), and ACC obtained after $30 \mathrm{~s}$ treatment time (30s).

For further investigating the crystallinity changes during the cellulose dissolution and ACC production, XRD patterns were recorded and then fitted (XRD diffraction patterns and fitting are presented in Figure S3 in Supporting information). The amount of amorphous cellulose remains nearly at the same level in the original pulp and ACCs, but the crystalline parts changed from $67 \%$ to around $25 \%$ and from $20 \%$ to $60 \%$ for cellulose I and cellulose II, respectively (Table 1 ). The ACCs showed a high preferred orientation of ca. $30 \%$ in cellulose II along the (1) 0 )-plane. These results support the findings of the ATR-IR spectroscopy for the ACCs. It has been reported that despite being natural cellulose, dissolving pulp can contain both cellulose I and cellulose II crystalline structure[41,42], which was also observed here. Presence of small amount of cellulose I in regenerated pulp might be due to the presence of minimal amount non-dissolved highly crystalline cellulose, which could not be observed with microscope (possible nanosized materials). The dissolution time did not have a visible influence on the crystallinity of the ACCs. Previous studies with $\mathrm{NaOH}$-urea showed lower conversion rate of cellulose I to cellulose II with similar treatment times (after treatment, cellulose II content were around 30\%)[29]. On the other 
hand, similar cellulose II content (around 60\%) than in current study were observed using long treatment time (20 minutes) with $\mathrm{NaOH}-u$ rea.[34] Higher decree of amorphous or non-crystalline cellulose in regenerated cellulose compared to ACCs is mostly likely due to the use of ethanol as coagulation bath for fully dissolved cellulose, whereas aqueous $\mathrm{HCl}$ solution was used during the production of ACCs.[40]

Table 1. Relative amounts of cellulose I, cellulose II, and amorphous cellulose of original dissolving pulp (Ref), regenerated cellulose (Reg), and ACCs with different treatment times ( $\eta$ is the degree of the preferred orientation and $r$ is the March-Dollase parameter).

\begin{tabular}{|c|c|c|c|c|c|}
\hline \multirow[b]{2}{*}{ Sample } & \multirow[b]{2}{*}{$\mathbf{r}$} & \multirow{2}{*}{$\begin{array}{l}\eta_{P O} \\
(\%)\end{array}$} & \multirow{2}{*}{$\begin{array}{c}\text { Amorphous } \\
(\%)\end{array}$} & \multirow{2}{*}{ Cellulose I (\%) } & \multirow{2}{*}{$\begin{array}{c}\text { Cellulose II } \\
\text { (\%) }\end{array}$} \\
\hline & & & & & \\
\hline Ref & 1 & 0 & $13.2 \pm 0.5$ & $67.1 \pm 0.7$ & $19.6 \pm 0.8$ \\
\hline Reg & 1 & 0 & $24.2 \pm 0.8$ & $18.0 \pm 1.5$ & $57.7 \pm 1.2$ \\
\hline $30 \mathrm{~s}$ & 0.6 & 28.6 & $10.8 \pm 0.3$ & $33.0 \pm 0.5$ & $56.2 \pm 0.5$ \\
\hline $60 \mathrm{~s}$ & 0.5 & 37.8 & $8.7 \pm 0.3$ & $29.8 \pm 0.4$ & $61.5 \pm 0.5$ \\
\hline $120 \mathrm{~s}$ & 0.6 & 28.6 & $11.1 \pm 0.4$ & $19.6 \pm 0.6$ & $69.3 \pm 0.6$ \\
\hline $240 \mathrm{~s}$ & 0.6 & 28.6 & $8.1 \pm 0.5$ & $29.3 \pm 0.8$ & $62.6 \pm 0.7$ \\
\hline
\end{tabular}

SEM images showed partial dissolution of fibers on the surface of the ACCs (Figure 4). Some small fragments of cellulose fibers were observed, but most of the ACCs exhibited an even and slightly rough surface pattern. Interestingly, it was noted that a longer dissolution time led to a slightly more uneven surface structure. This might indicate that a longer dissolution time leads to release of the cellulose into solvent, causing erosion of the surface. Some of the dissolved cellulose might also be lost during the coagulation and washing of the sample. 


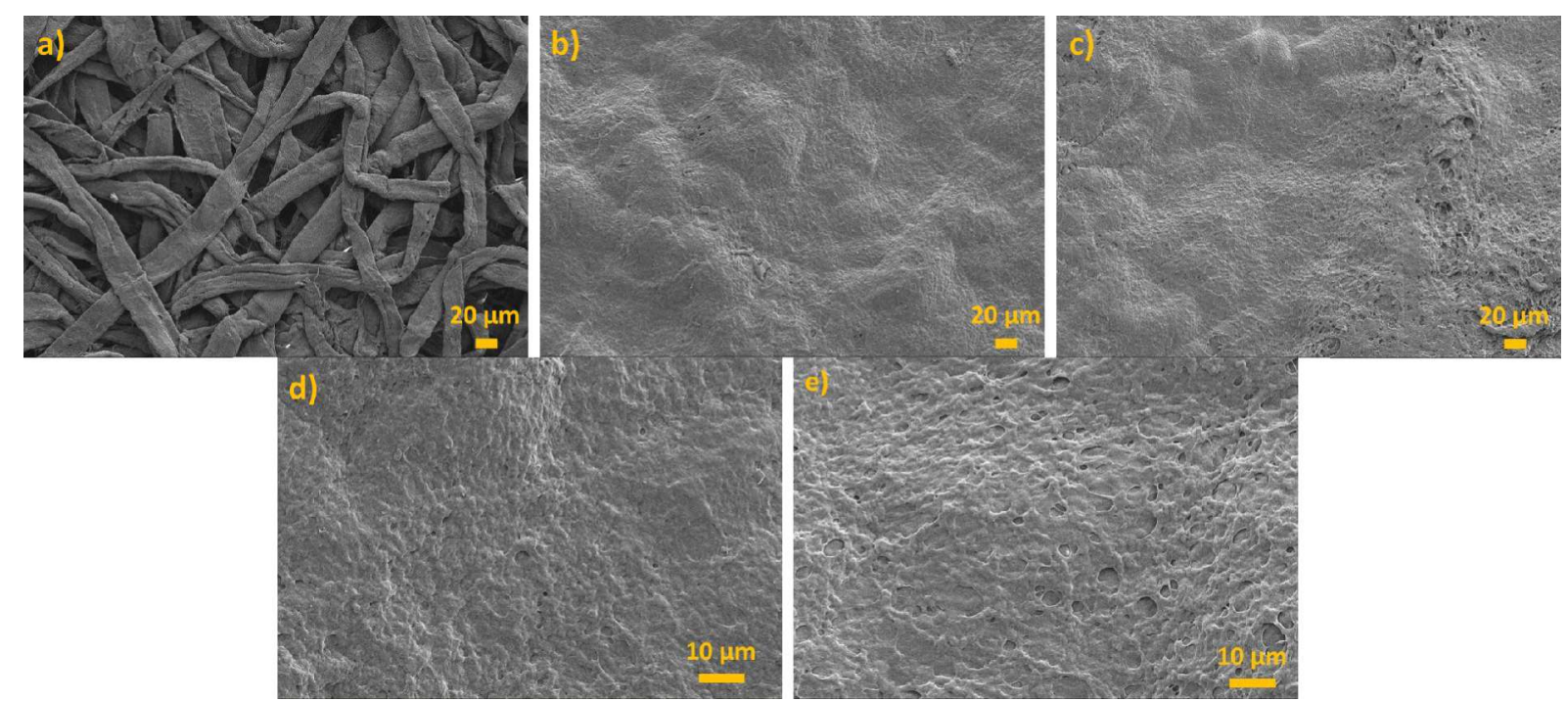

Figure 4. SEM surface images of a) reference cellulose sheet, ACCs after b) 30, c) 60, d) 120, and e) $180 \mathrm{~s}$ dissolution times.

Cross-sectional images of the ruptured reference cellulose sheet showed completely fibrous structure, with random pulling of fibers after breakage in tensile testing (Figure 5), whereas clear cutting of the ACC was obtained after tensile testing. The images in higher magnification showed that, in addition to the dissolved fibers on the surface, most of the inner fibers were also welded together and only small fiber fragments or fibers parts with sub-micron diameters were present. This further confirms the efficient solvent welding ability of TEAOH. 

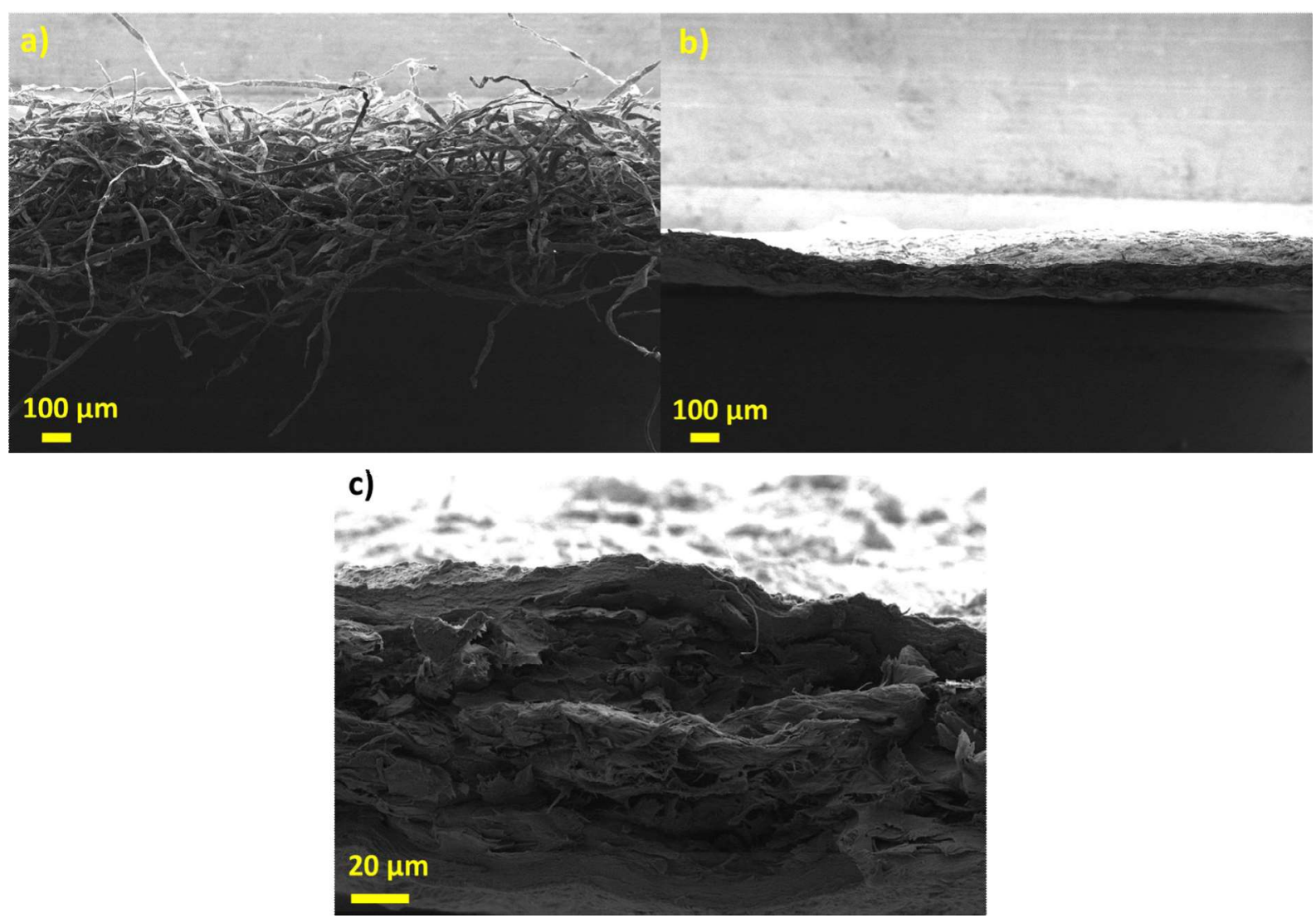

Figure 5. SEM images of cross-section of tensile test samples after rupture: a) reference cellulose sheet and b) and c) ACC after $30 \mathrm{~s}$ treatment time at different magnification.

The tensile strength of the dissolving pulp sheet increased from 3.3 to $57.8 \mathrm{MPa}$ only after $30 \mathrm{~s}$ treatment time (Table 2, average stress-to-strain curves are presented in Figure 6). In addition, the elastic modulus increased around tenfold, from $0.5 \mathrm{GPa}$ to $5.1 \mathrm{GPa}$, whereas the elongation at break remained at the same level. This demonstrates the efficient and rapid solvent welding properties of TEAOH to produce strong and stiff ACCs. There were no significant differences between the tensile strengths of ACCs when the treatment time was increased from 30 to $180 \mathrm{~s}$.

The tensile properties of the ACCs obtained in the current study are generally lower compared to several reported in literature.[2] However, very high tensile strength (several hundred MPa) are obtained in the longitudinal direction of the samples where fibers were pre-aligned before 
ACC production.[23] In addition, tensile strength over $100 \mathrm{MPa}$ could be obtained even from random-orientated fibers using $\mathrm{DMAc} / \mathrm{LiCl}$ at $20^{\circ} \mathrm{C},[43]$ which is higher than in current study were the preparation of cellulose sheets also results in the random alignment of fibers. However, the relative improvement of tensile strength of the cellulose sheet during the ACC production using TEAOH was very high. For example, the use of ionic liquids to produce ACC from filter paper increased the tensile strength to around seven times the initial value after $160 \mathrm{~min}$ of treatment time at $80{ }^{\circ} \mathrm{C}$.[44] Using 12 hours dissolution time in aqueous polyethylene glygol$\mathrm{NaOH}$ at $-12{ }^{\circ} \mathrm{C}$, tensile strength of filter paper improved around three times from the initial value [45], whereas around four times increase in the tensile strength has been observed using DMAc/ $\mathrm{LiCl}$ with six hours at $30^{\circ} \mathrm{C}$.[38] In current study, over 17 times higher tensile strength was obtained only after $30 \mathrm{~s}$ treatment time using TEAOH at room temperature. Previously, similar results were reported using $\mathrm{NaOH}$-urea solution to produce ACCs (increase in tensile strength from 3.4 to $48.8 \mathrm{MPa}$ after $30 \mathrm{~s}$ treatment times).[29] 
Table 2. Effect of the treatment time (s, seconds) and hot-pressing (hp) on the properties of ACCs (error represents standard deviation).

\begin{tabular}{ccccc}
\hline Sample & $\begin{array}{c}\text { Elastic modulus } \\
\text { (GPa) }\end{array}$ & $\begin{array}{c}\text { Elongation at break } \\
\mathbf{( \% )}\end{array}$ & $\begin{array}{c}\text { Stress at break } \\
\text { (MPa) }\end{array}$ & Thickness $(\boldsymbol{\mu m})$ \\
\hline Reference & $0.5 \pm 0.01$ & $1.8 \pm 0.2$ & $3.3 \pm 0.2$ & $179 \pm 1$ \\
$30 \mathrm{~s}$ & $5.1 \pm 0.6$ & $1.7 \pm 0.2$ & $55.0 \pm 2.7$ & $122 \pm 1$ \\
$60 \mathrm{~s}$ & $4.4 \pm 0.7$ & $2.1 \pm 0.3$ & $51.7 \pm 4.9$ & $134 \pm 1$ \\
$120 \mathrm{~s}$ & $4.7 \pm 0.9$ & $1.8 \pm 0.5$ & $53.5 \pm 5.9$ & $132 \pm 1$ \\
$180 \mathrm{~s}$ & $4.6 \pm 1.1$ & $1.6 \pm 0.3$ & $48.6 \pm 6.7$ & $142 \pm 2$ \\
$30 \mathrm{~s}, \mathrm{hp}$ & $7.2 \pm 0.4$ & $2.0 \pm 0.2$ & $75.0 \pm 4.5$ & $99 \pm 0$ \\
$60 \mathrm{~s}, \mathrm{hp}$ & $6.7 \pm 0.7$ & $2.2 \pm 0.1$ & $75.9 \pm 8.9$ & $111 \pm 1$ \\
$120 \mathrm{~s}, \mathrm{hp}$ & $6.8 \pm 0.6$ & $2.3 \pm 0.0$ & $72.5 \pm 4.7$ & $91 \pm 0$ \\
$180 \mathrm{~s}, \mathrm{hp}$ & $6.8 \pm 0.6$ & $2.2 \pm 0.1$ & $76.7 \pm 7.8$ & $109 \pm 0$ \\
\hline
\end{tabular}
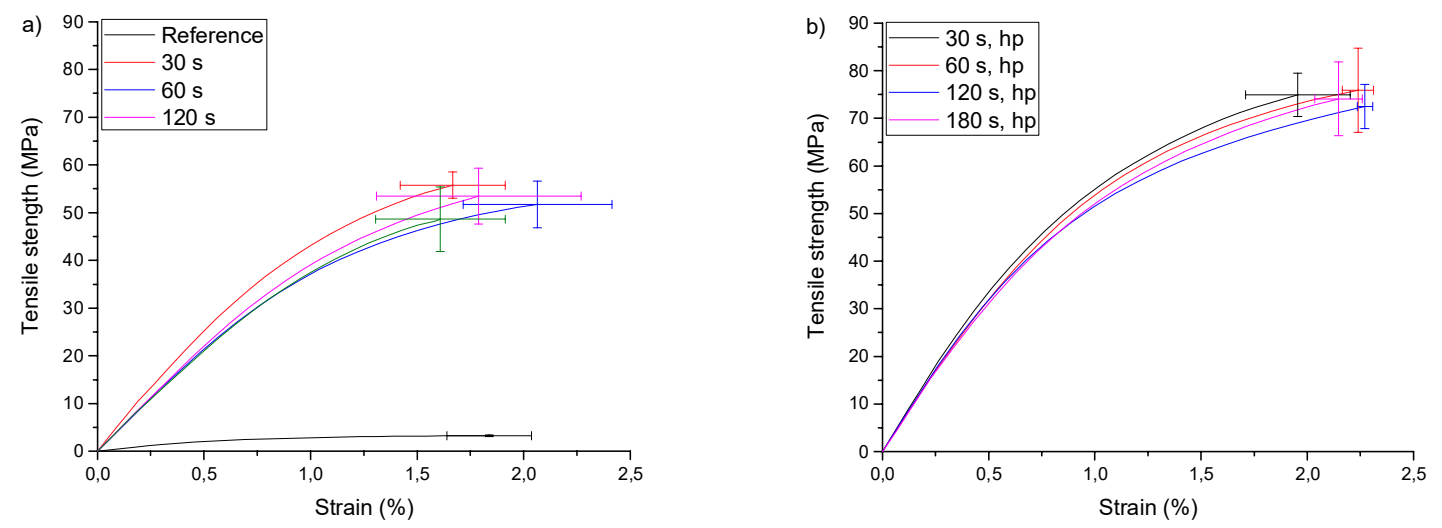
Figure 6. Average stress-strain curves of cellulose sheet (reference) and ACCs after a) different treatment times (s, second) and b) high-pressure aided hot-pressing (hp) (error bars represents standard deviation).

The tensile properties of AACs were further improved by high-pressure aided hot-pressing of the washed samples. For instance, the tensile strength of the ACC after $30 \mathrm{~s}$ treatment time increased to $75 \mathrm{MPa}$ (36\% higher compared to samples prepared by hand sheet grill drying) when high-pressure-aided hot-pressing was utilized (Table 2). It has been previously shown that use of high pressure can increase the mechanical properties of the ACCs.[46] The increase in the tensile properties was most likely due to the formation of a more compacted structure, which was observed as a decrease in the thicknesses of the samples (from 122 to $99 \mu \mathrm{m}$ when $30 \mathrm{~s}$ dissolution time was applied).

\section{Conclusions}

Dissolving softwood pulp could readily be dissolved in an aqueous TEAOH solution at room temperature within three hours. Up to $3 \mathrm{wt} \%$ dissolved cellulose solution could be attained using simple magnetic stirring, whereas a higher concentration might require more intense mixing. No hydrolysis of cellulose occurred as the DP of cellulose was observed to remain intact after dissolution and regeneration. TEAOH could be used to produce ACCs at room temperature by solvent welding of the cellulose fiber sheets. Over 17 times higher tensile strength was obtained only after $30 \mathrm{~s}$ dissolution time, demonstrating the efficient solvent capacity of TEAOH. The high-pressure aided hot-pressing of ACCs prior to drying could be used to further increase the tensile properties up to 22-fold (compared to original cellulose sheets) with $30 \mathrm{~s}$ treatment time. This study demonstrated a sustainable procedure for both cellulose dissolution and rapid 
production of strong ACCs using methodologies that could be feasible even at the industrial scale.

\section{Acknowledgements}

Joni Vasara and Jani Österlund are thanked for their contribution to the experimental part of the study. Ilkka Miinalainen, Marcin Selent, and Elisa Wikkala acknowledged for performing SEM, XRD and limiting viscosity measurements.

\section{References}

[1] Suhas, V.K. Gupta, P.J.M. Carrott, R. Singh, M. Chaudhary, S. Kushwaha, Cellulose: A review as natural, modified and activated carbon adsorbent, Bioresour. Technol. 216 (2016) 1066-1076. doi:10.1016/j.biortech.2016.05.106.

[2] T. Huber, J. Müssig, O. Curnow, S. Pang, S. Bickerton, M.P. Staiger, A critical review of all-cellulose composites, J. Mater. Sci. 47 (2011) 1171-1186. doi:10.1007/s10853-0115774-3.

[3] S.C. Fox, B. Li, D. Xu, K.J. Edgar, Regioselective Esterification and Etherification of Cellulose: A Review, Biomacromolecules. 12 (2011) 1956-1972. doi:10.1021/bm200260d.

[4] M. Isik, H. Sardon, D. Mecerreyes, Ionic Liquids and Cellulose: Dissolution, Chemical Modification and Preparation of New Cellulosic Materials, Int. J. Mol. Sci. 15 (2014) 11922-11940. doi:10.3390/ijms150711922.

[5] J. Pang, X. Liu, J. Yang, F. Lu, B. Wang, F. Xu, M. Ma, X. Zhang, Synthesis of Highly Polymerized Water-soluble Cellulose Acetate by the Side Reaction in Carboxylate Ionic Liquid 1-ethyl-3-methylimidazolium Acetate, Sci. Rep. $6 \quad$ (2016) 33725. doi:10.1038/srep33725.

[6] J.A. Sirviö, J.P. Heiskanen, Synthesis of Alkaline-Soluble Cellulose Methyl Carbamate Using a Reactive Deep Eutectic Solvent, ChemSusChem. 10 (2017) 455-460. doi:10.1002/cssc. 201601270 .

[7] H.-P. Fink, J. Ganster, A. Lehmann, Progress in cellulose shaping: 20 years industrial case studies at Fraunhofer IAP, Cellulose. 21 (2013) 31-51. doi:10.1007/s10570-013-0137-7.

[8] T. Rosenau, A. Potthast, I. Adorjan, A. Hofinger, H. Sixta, H. Firgo, P. Kosma, Cellulose solutions in N-methylmorpholine-N-oxide (NMMO) - degradation processes and stabilizers, Cellulose. 9 (n.d.) 283-291. doi:10.1023/A:1021127423041.

[9] T.R. Dawsey, C.L. McCormick, The Lithium Chloride/Dimethylacetamide Solvent for Cellulose: A Literature Review, J. Macromol. Sci. Part C. 30 (1990) 405-440. doi:10.1080/07366579008050914.

[10] S. Fischer, H. Leipner, K. Thümmler, E. Brendler, J. Peters, Inorganic Molten Salts as Solvents for Cellulose, Cellulose. 10 (2003) 227-236. doi:10.1023/A:1025128028462.

[11] A. Pinkert, K.N. Marsh, S. Pang, M.P. Staiger, Ionic Liquids and Their Interaction with Cellulose, Chem. Rev. 109 (2009) 6712-6728. doi:10.1021/cr9001947. 
[12] H. Ren, Q. Wang, S. Guo, D. Zhao, C. Chen, The role and potential of morpholinium-based ionic liquids in dissolution of cellulose, Eur. Polym. J. 92 (2017) 204-212. doi:10.1016/j.eurpolymj.2017.05.011.

[13] R. Rinaldi, Instantaneous dissolution of cellulose in organic electrolyte solutions, Chem. Commun. 47 (2010) 511-513. doi:10.1039/C0CC02421J.

[14] J. Cai, L. Zhang, Rapid Dissolution of Cellulose in LiOH/Urea and NaOH/Urea Aqueous Solutions, Macromol. Biosci. 5 (2005) 539-548. doi:10.1002/mabi.200400222.

[15] L. Zhang, D. Ruan, S. Gao, Dissolution and regeneration of cellulose in NaOH/thiourea aqueous solution, J. Polym. Sci. Part B Polym. Phys. 40 (2002) 1521-1529. doi:10.1002/polb.10215.

[16] S. Grönqvist, T. Kamppuri, T. Maloney, M. Vehviläinen, T. Liitiä, A. Suurnäkki, Enhanced pre-treatment of cellulose pulp prior to dissolution into $\mathrm{NaOH} / \mathrm{ZnO}$, Cellulose. 22 (2015) 3981-3990. doi:10.1007/s10570-015-0742-8.

[17] S. Schmidt, T. Liebert, T. Heinze, Synthesis of soluble cellulose tosylates in an eco-friendly medium, Green Chem. 16 (2014) 1941-1946. doi:10.1039/C3GC41994K.

[18] N.L. Moigne, P. Navard, Dissolution mechanisms of wood cellulose fibres in $\mathrm{NaOH}$-water, Cellulose. 17 (2009) 31-45. doi:10.1007/s10570-009-9370-5.

[19] M. Abe, K. Kuroda, H. Ohno, Maintenance-Free Cellulose Solvents Based on Onium Hydroxides, ACS Sustain. Chem. Eng. 3 (2015) 1771-1776. doi:10.1021/acssuschemeng.5b00303.

[20] C. Zhong, C. Wang, F. Wang, H. Jia, P. Wei, Y. Zhao, Application of tetra-nmethylammonium hydroxide on cellulose dissolution and isolation from sugarcane bagasse, Carbohydr. Polym. 136 (2016) 979-987. doi:10.1016/j.carbpol.2015.10.001.

[21] W. Wei, F. Meng, Y. Cui, M. Jiang, Z. Zhou, Room temperature dissolution of cellulose in tetra-butylammonium hydroxide aqueous solvent through adjustment of solvent amphiphilicity, Cellulose. (2016) 1-11. doi:10.1007/s10570-016-1113-9.

[22] C. Zhong, C. Wang, F. Huang, H. Jia, P. Wei, Wheat straw cellulose dissolution and isolation by tetra-n-butylammonium hydroxide, Carbohydr. Polym. 94 (2013) 38-45. doi:10.1016/j.carbpol.2013.01.043.

[23] N. Soykeabkaew, N. Arimoto, T. Nishino, T. Peijs, All-cellulose composites by surface selective dissolution of aligned ligno-cellulosic fibres, Compos. Sci. Technol. 68 (2008) 2201-2207. doi:10.1016/j.compscitech.2008.03.023.

[24] T. Nishino, I. Matsuda, K. Hirao, All-Cellulose Composite, Macromolecules. 37 (2004) 7683-7687. doi:10.1021/ma049300h.

[25] H. Yousefi, M. Mashkour, R. Yousefi, Direct solvent nanowelding of cellulose fibers to make all-cellulose nanocomposite, Cellulose. 22 (2015) 1189-1200. doi:10.1007/s10570015-0579-1.

[26] S. Ouajai, R.A. Shanks, Preparation, structure and mechanical properties of all-hemp cellulose biocomposites, Compos. Sci. Technol. 69 (2009) 2119-2126. doi:10.1016/j.compscitech.2009.05.005.

[27] Q. Zhao, R.C.M. Yam, B. Zhang, Y. Yang, X. Cheng, R.K.Y. Li, Novel all-cellulose ecocomposites prepared in ionic liquids, Cellulose. 16 (2009) 217-226. doi:10.1007/s10570-008-9251-3.

[28] H. Qi, J. Cai, L. Zhang, S. Kuga, Properties of Films Composed of Cellulose Nanowhiskers and a Cellulose Matrix Regenerated from Alkali/Urea Solution, Biomacromolecules. 10 (2009) 1597-1602. doi:10.1021/bm9001975. 
[29] P. Piltonen, N.C. Hildebrandt, B. Westerlind, J.-P. Valkama, T. Tervahartiala, M. Illikainen, Green and efficient method for preparing all-cellulose composites with $\mathrm{NaOH} / \mathrm{urea}$ solvent, Compos. Sci. Technol. 135 (2016) 153-158. doi:10.1016/j.compscitech.2016.09.022.

[30] J. Sirvio, U. Hyvakko, H. Liimatainen, J. Niinimaki, O. Hormi, Periodate oxidation of cellulose at elevated temperatures using metal salts as cellulose activators, Carbohydr. Polym. 83 (2011) 1293-1297. doi:10.1016/j.carbpol.2010.09.036.

[31] ISO 5263-1:2004 - Pulps -- Laboratory wet disintegration -- Part 1: Disintegration of chemical pulps, (n.d.). https://www.iso.org/standard/37893.html (accessed July 31, 2017).

[32] ISO 5269-1:1998 - Pulps -- Preparation of laboratory sheets for physical testing -- Part 1: Conventional sheet-former method, (n.d.). https://www.iso.org/standard/11264.html (accessed March 27, 2017).

[33] S. Nam, A.D. French, B.D. Condon, M. Concha, Segal crystallinity index revisited by the simulation of X-ray diffraction patterns of cotton cellulose I $\beta$ and cellulose II, Carbohydr. Polym. 135 (2016) 1-9. doi:10.1016/j.carbpol.2015.08.035.

[34] B. Duchemin, D.L. Corre, N. Leray, A. Dufresne, M.P. Staiger, All-cellulose composites based on microfibrillated cellulose and filter paper via a $\mathrm{NaOH}$-urea solvent system, Cellulose. 23 (2015) 593-609. doi:10.1007/s10570-015-0835-4.

[35] E. Zolotoyabko, Determination of the degree of preferred orientation within the MarchDollase approach, J. Appl. Crystallogr. 42 (2009) 513-518. doi:10.1107/S0021889809013727.

[36] P.L. Nasatto, F. Pignon, J.L.M. Silveira, M.E.R. Duarte, M.D. Noseda, M. Rinaudo, Methylcellulose, a Cellulose Derivative with Original Physical Properties and Extended Applications, Polymers. 7 (2015) 777-803. doi:10.3390/polym7050777.

[37] T. Ema, T. Komiyama, S. Sunami, T. Sakai, Synergistic effect of quaternary ammonium hydroxide and crown ether on the rapid and clear dissolution of cellulose at room temperature, RSC Adv. 4 (2014) 2523-2525. doi:10.1039/C3RA45888A.

[38] T. Nishino, N. Arimoto, All-Cellulose Composite Prepared by Selective Dissolving of Fiber Surface, Biomacromolecules. 8 (2007) 2712-2716. doi:10.1021/bm0703416.

[39] N. Isobe, U.-J. Kim, S. Kimura, M. Wada, S. Kuga, Internal surface polarity of regenerated cellulose gel depends on the species used as coagulant, J. Colloid Interface Sci. 359 (2011) 194-201. doi:10.1016/j.jcis.2011.03.038.

[40] Å. Östlund, A. Idström, C. Olsson, P.T. Larsson, L. Nordstierna, Modification of crystallinity and pore size distribution in coagulated cellulose films, Cellulose. 20 (2013) 1657-1667. doi:10.1007/s10570-013-9982-7.

[41] Estimation of Cellulose I and II in Cellulosic Samples by Principal Component Analysis of 13C-CP/MAS-NMR-Spectra: Holzforschung - International Journal of the Biology, Chemistry, Physics and Technology of Wood, (1995). https://www.degruyter.com/view/j/hfsg.1995.49.issue2/hfsg.1995.49.2.119/hfsg.1995.49.2.119.xml (accessed July 31, 2017).

[42] H. Kumar, L.P. Christopher, Recent trends and developments in dissolving pulp production and application, Cellulose. 24 (2017) 2347-2365. doi:10.1007/s10570-017-1285-y.

[43] W. Gindl, T. Schöberl, J. Keckes, Structure and properties of a pulp fibre-reinforced composite with regenerated cellulose matrix, Appl. Phys. A. 83 (2006) 19-22. doi:10.1007/s00339-005-3451-6. 
[44] B.J.C. Duchemin, A.P. Mathew, K. Oksman, All-cellulose composites by partial dissolution in the ionic liquid 1-butyl-3-methylimidazolium chloride, Compos. Part Appl. Sci. Manuf. 40 (2009) 2031-2037. doi:10.1016/j.compositesa.2009.09.013.

[45] D. Han, L. Yan, Preparation of all-cellulose composite by selective dissolving of cellulose surface in PEG/NaOH aqueous solution, Carbohydr. Polym. 79 (2010) 614-619. doi:10.1016/j.carbpol.2009.09.008.

[46] M. Shibata, N. Teramoto, T. Nakamura, Y. Saitoh, All-cellulose and all-wood composites by partial dissolution of cotton fabric and wood in ionic liquid, Carbohydr. Polym. 98 (2013) 1532-1539. doi:10.1016/j.carbpol.2013.07.062. 